\title{
Developing Computationally Efficient Property Calculations for High-Pressure, Multi- Component Droplet Vaporization
}

\author{
Michael Hayes ${ }^{1}$, Simcha Singer ${ }^{2}$ \\ ${ }^{1}$ Student (Mechanical Engineering, Marquette University) Milwaukee, WI \\ ${ }_{2}^{2}$ Professor (Mechanical Engineering, Marquette University) Milwaukee, WI
}

\begin{abstract}
The combustion of liquid fuels such as gasoline, diesel, and jet fuels often occurs at elevated pressures. The complex composition of these hydrocarbon fuels makes modeling them a time-consuming process using traditional discrete component models (DCMs); continuous thermodynamic models (CTMs) are more computationally efficient. This paper utilizes high-pressure property calculations to improve upon the accuracy of an existing CTM in depicting the vaporization of liquid fuel droplets at high pressures. Increased model fidelity in replicating DCM results is accomplished by depicting these properties as functions of temperature, pressure, and a chosen distribution variable. This addition leads to high simulation accuracy at elevated pressures while maintaining the computational efficiency of CTMs.
\end{abstract}

\section{Introduction}

The vaporization behavior of fuel droplets is an important area of research in the field of combustion. Liquid fuels such as gasoline, diesel, and jet fuels play important roles in transportation and industry. Furthermore, understanding the behavior of these substances is crucial to ensuring their responsible use. While simple hydrocarbon mixtures can be modeled with relative ease, describing and predicting the behavior of more complex fuels is impeded by two major problems: fuels like gasoline are comprised of dozens if not hundreds of different hydrocarbons (Lovestead et al., 2016), and combustion reactions involving these fuels often occur at high pressures. Both factors complicate the calculations necessary to adequately describe fuel behavior.

While it is theoretically possible to simulate a multi-component fuel's combustion using a large mixture of hydrocarbon species in what is known as a discrete component model (DCM) (Torres et al., 2003), such a procedure would incur an impractically long computational runtime. A possible remedy to this problem is the utilization of a "surrogate" fuel that can mimic the properties of the actual fuel it represents while being comprised of just a few species. However, surrogate fuels developed to mimic a real fuel's combustion behavior are often unable to represent the liquid fuel's vaporization behavior and physical properties. This paper focuses on a recently developed hybrid approach: the use of a continuous thermodynamic model (CTM) to model fuel droplet vaporization, which is then lumped to produce a chemical surrogate that mimics the fuel's combustion behavior (Cooney \& Singer, 2019). In particular, the CTM utilizes the Coupled Algebraic-Direct Quadrature Method of Moments to model the droplet vaporization behavior in a computationally efficient and accurate manner.

For the CTM, the multicomponent fuel's physical and thermodynamic properties must be described as functions of a distribution variable (Tamim \& Hallett, 1995). Many such models factor in the temperature dependence of properties such as thermal conductivity and viscosity

\footnotetext{
This research would not have been possible without the generous support of the National Space Grant College and Fellowship Program and the Wisconsin Space Grant Consortium. In addition, Marquette University's assistance in the form of a Summer Undergraduate Research Fellowship further contributed to the success of this project.
} 
over the duration of a droplet's vaporization. A deficiency exists, however, in considering the effect of pressure on these properties. Yan and Aggarwal (2006) and Zhang and Kong (2011) provide two examples of how correction calculations can be utilized to improve both the accuracy of properties and overall model performance. Neither of these models, though, applies high-pressure property corrections to CTM calculations for multi-component mixtures. The goal of this research is to develop high-pressure property calculations for a computationally efficient, quadrature-based moment method for multicomponent droplet vaporization.

\section{Background and Methods}

2.1 Droplet vaporization model. The droplet vaporization CTM used in this work is based on the one utilized by Cooney and Singer (2019). The mathematics of this quadraturebased moment method will not be discussed here, aside from the necessary references to property calculations which must be obtained as a function of a distribution variable. A comparative DCM (which is computationally inefficient, as it contains every discrete species) will also be used to evaluate the accuracy of the CTM.

2.2 Low-pressure property calculations. At low pressures, the thermal conductivity and viscosity of both liquids and gases can be approximated as functions of temperature alone. Based on the data of Yaws $(2012 ; 2013 ; 2014)$, equations can be represented as shown:

$$
\begin{gathered}
k_{g}=A+B T_{g}+C T_{g}^{2}+D T_{g}^{3} \\
k_{l}=A+B T_{g}+C T_{g}^{2} \\
\mu_{g}=A+B T_{g}+C T_{g}^{2}+D T_{g}^{3} \\
\log \left(\mu_{l}\right)=A+\frac{B}{T_{l}}+C T_{l}+D T_{l}^{2}
\end{gathered}
$$

Within these equations $k$ represents thermal conductivity, $\mu$ for viscosity, $T$ for temperature, and $A, B, C$, and $D$ are correlation coefficients unique to each component species. Gas diffusivity, while not dependent solely on temperature, can be described at low pressures by Fuller et al.'s (1966) equation:

$$
D_{g}=\frac{(0.001) * T_{g}^{1.75}\left(\frac{1}{M W_{\text {fuel }}}-\frac{1}{M W_{\text {air }}}\right)^{\frac{1}{2}}}{P_{\infty}\left[\left(v_{\text {fuel }}\right)^{\frac{1}{3}}+\left(v_{\text {air }}\right)^{\frac{1}{3}}\right]^{2}}
$$

where the pressure, molecular weight, and atomic diffusion volume of the mixture components are shown as $P, M W$, and $v$, respectively. These equations, while appropriate at low pressures, neglect to take into account the effect of elevated pressures on property values.

2.3 High-pressure property calculations. In order to produce accurate property values at elevated pressures, supplementary or replacement equations are required. Reid et al. (1987) 
provide a comprehensive review of different possible methods, and five high-pressure calculations were identified for use in determining the property values chosen for this paper. All of these calculations are summarized in detail in Reid's authoritative text. The first, concerning gas thermal conductivity, comes from Ely and Hanley (1983). Due to the length of the procedure, the full calculations will not be included here. Instead, the high-level equation provided by Reid et al. is shown:

$$
k_{g}=k^{* *}+\frac{\mu^{*}}{M^{\prime}}(1.32)\left(C_{v}-\frac{3 R}{2}\right)
$$

The correction for liquid thermal conductivity comes from the method of Latini and Baroncini (1983). In order to simplify the calculation, it was assumed that all components considered were saturated hydrocarbons. After rearranging, the following equation can be found:

$$
k_{l}=k_{l 0}+\frac{0.0673 * P_{r} *\left(1-T_{r}\right)^{0.38}}{M^{0.84} * T_{r}^{\frac{1}{6}}}
$$

For calculating gas viscosity, the method of Jossi et al. (1962) was implemented. For the sake of brevity, only the high-level equation is shown here:

$$
\mu_{g}=\frac{\left[\left(1.0230+0.23364 \rho_{r}+0.58533 \rho_{r}^{2}+0.40758 \rho_{r}^{3}+0.093324 \rho_{r}^{4}\right)^{4}-1\right]}{\xi_{T}}
$$

Similarly, Lucas' (1981) method for calculating liquid viscosity is shown below:

$$
\mu_{l}=\mu_{l 0} * \frac{1+D\left(\frac{\Delta P_{r}}{2.118}\right)^{A}}{1+C \omega \Delta P_{r}}
$$

The final property considered here is the diffusivity between species in the gas state and other species surrounding the droplet. Takahashi's (1974) calculation for high pressures, which utilizes tabulated values, was implemented to determine a corrected diffusivity value:

$$
D_{A B}=\frac{\left(D_{A B} P\right)^{+} f\left(T_{r}, P_{r}\right)}{P}
$$

2.4 Comparison to experimental data. Having identified these five corrections, it was next necessary to ensure their veracity and the validity of the droplet model into which they are incorporated. To accomplish this, both the existing DCM model and a DCM model featuring high-pressure property calculations were compared to experimental data for n-heptane droplet vaporization at elevated pressures (Nomura et al., 1996). As Figure 1 shows, the corrected model provides a more accurate prediction of droplet behavior than its low-pressure counterpart. The corrected model performs better at a variety of temperatures as well, suggesting that the new 
model retains its temperature dependence while still implementing accurate calculations at high pressures.
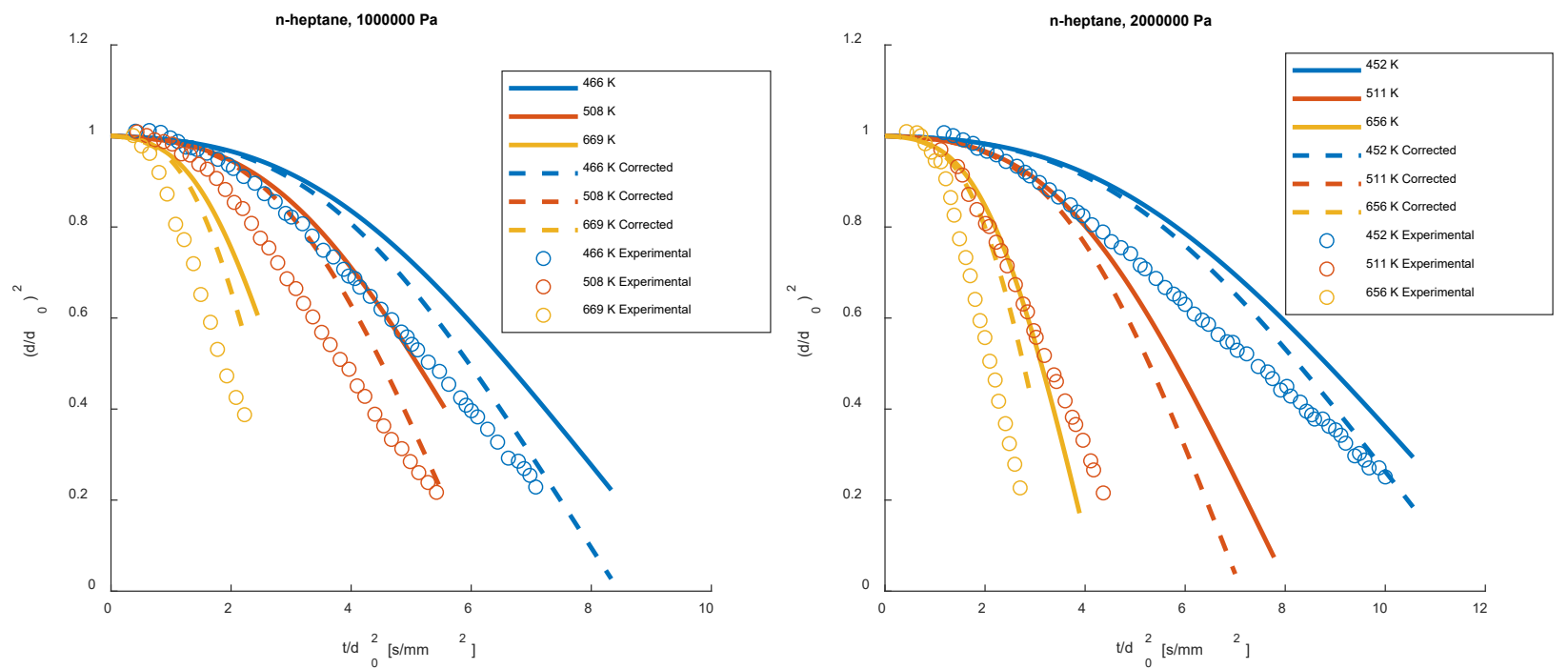

Fig 1: Comparison of model performance to experimental data

It is important to note, however, that discrepancies still exist between the corrected model and the actual experimental data. While high-pressure property calculations provide an increase in accuracy, it is likely that other high-pressure vaporization physics need to be reevaluated as well in order to ensure that they too properly describe combustion behavior at elevated pressures. Such additional changes, though, lie beyond the scope of this paper.

\subsection{Generation of pressure-}

temperature surfaces for CTM use. Having verified the improved accuracy of the highpressure property equations, the next step was to apply them to the continuous thermodynamic model. CTM's calculate properties based on a function of a distribution variable. The distribution variable is often chosen as the boiling point at atmospheric pressure or molecular weight, but in this model the species are arranged (via multi-objective optimization) to create a continuous function of a purely mathematical variable, $I$, that is able to correlate both physical properties and chemical functional groups (Cooney \& Singer, 2019). An example of this is shown in Figure 2, which illustrates the dependence of gas thermal conductivity on the distribution variable, $I$.

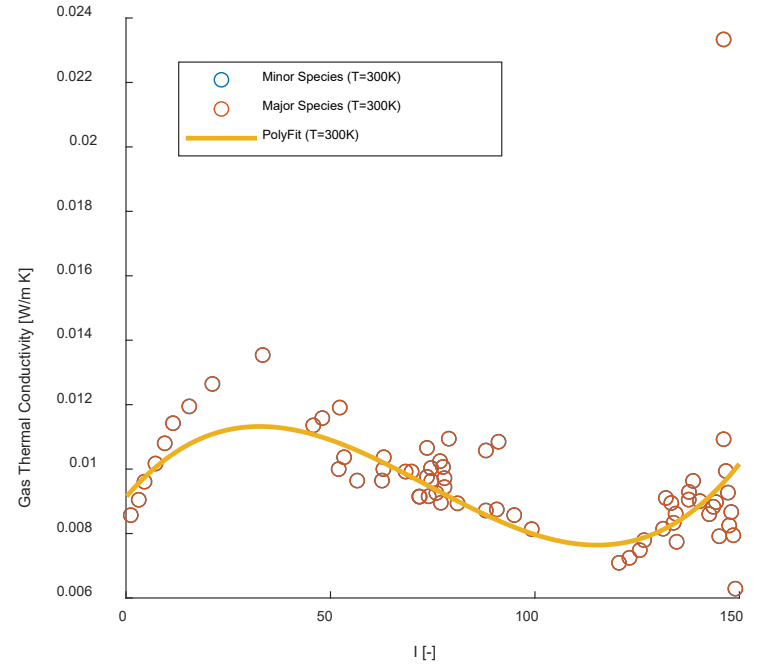

Figure 2: A continuous function describing gas thermal conductivity at $300 \mathrm{~K}$ and atmospheric pressure 
The property values (thermal conductivity, in the case of this example) can be determined simply by fitting a third order polynomial in $I$. In its most basic form, this polynomial is solely a function of the distribution variable and does not take into account the effects of additional factors such as temperature and pressure. Such a function can be most basically described by the equation below:

$$
k_{g}=A+B I+C I^{2}+D I^{3}
$$

Where $A, B, C$, and $D$ are fit coefficients and $I$ is the distribution variable. For a temperaturedependent property, such as the thermal conductivity shown in Figure 2, Cooney and Singer substituted functions of temperature as fit coefficients:

$$
\begin{aligned}
k_{g}=(a+b T & \left.+c T^{2}\right)+\left(d+e T+f T^{2}\right) I+\left(g+h T+i T^{2}\right) I^{2} \ldots \\
& +\left(j+k T+l T^{2}\right) I^{3}
\end{aligned}
$$

With this added functionality, the continuous distribution can account for changes to thermal conductivity with temperature. A quadratic fit best describes the behavior in this case; hence quadratic expressions are used to generate suitable coefficients for the equation's $I$ terms. This approach can be modified to include the effect of pressure as well. Below is the modified equation created for gas thermal conductivity:

$$
\begin{gathered}
k_{g}=\left(a_{0}+b_{0} P+c_{0} T+d_{0} P^{2}+e_{0} P T+f_{0} T^{2}+g_{0} P^{3}+h_{0} P^{2} T \ldots\right. \\
\left.\quad+i_{0} P T^{2}+j_{0} T^{3}\right) \ldots \\
+\left(a_{1}+b_{1} P+c_{1} T+d_{1} P^{2}+e_{1} P T+f_{1} T^{2}+g_{1} P^{3}+h_{1} P^{2} T \ldots\right. \\
\left.\quad+i_{1} P T^{2}+j_{1} T^{3}\right) I \ldots \\
+\left(a_{2}+b_{2} P+c_{2} T+d_{2} P^{2}+e_{2} P T+f_{2} T^{2}+g_{2} P^{3}+h_{2} P^{2} T \ldots\right. \\
\left.+i_{2} P T^{2}+j_{2} T^{3}\right) I^{2} \ldots \\
+\left(a_{3}+b_{3} P+c_{3} T+d_{3} P^{2}+e_{3} P T+f_{3} T^{2}+g_{3} P^{3}+h_{3} P^{2} T \ldots\right. \\
\left.+i_{3} P T^{2}+j_{3} T^{3}\right) I^{3}
\end{gathered}
$$

While containing many more terms, this expression retains the same essential structure of providing coefficients for the equation's $I$ terms. The reason for the increase in $P$ and $T$ coefficients is shown in Figure 3, which compares a plot of the first group from Equation 12 with the first group from Equation 13. Careful comparison shows that the two-dimensional plot, focused solely on temperature dependency and assumed to be at atmospheric pressure, can roughly fit into the surface shown to its right. The slight discrepancy between the two likely stems from the fact that the surface is generated based on the high-pressure equations - lower pressures can be more accurately described by simpler models.

Third-order functions were chosen for Equation 13 due to their accuracy in depicting the surface bounded by the points shown in Figure 3. This surfacing procedure was replicated for each of the properties elaborated on in this paper, except for gas diffusivity (which is calculated in a different manner). A variety of fits were evaluated to generate a surface that captured the most data points for each polynomial coefficient. 

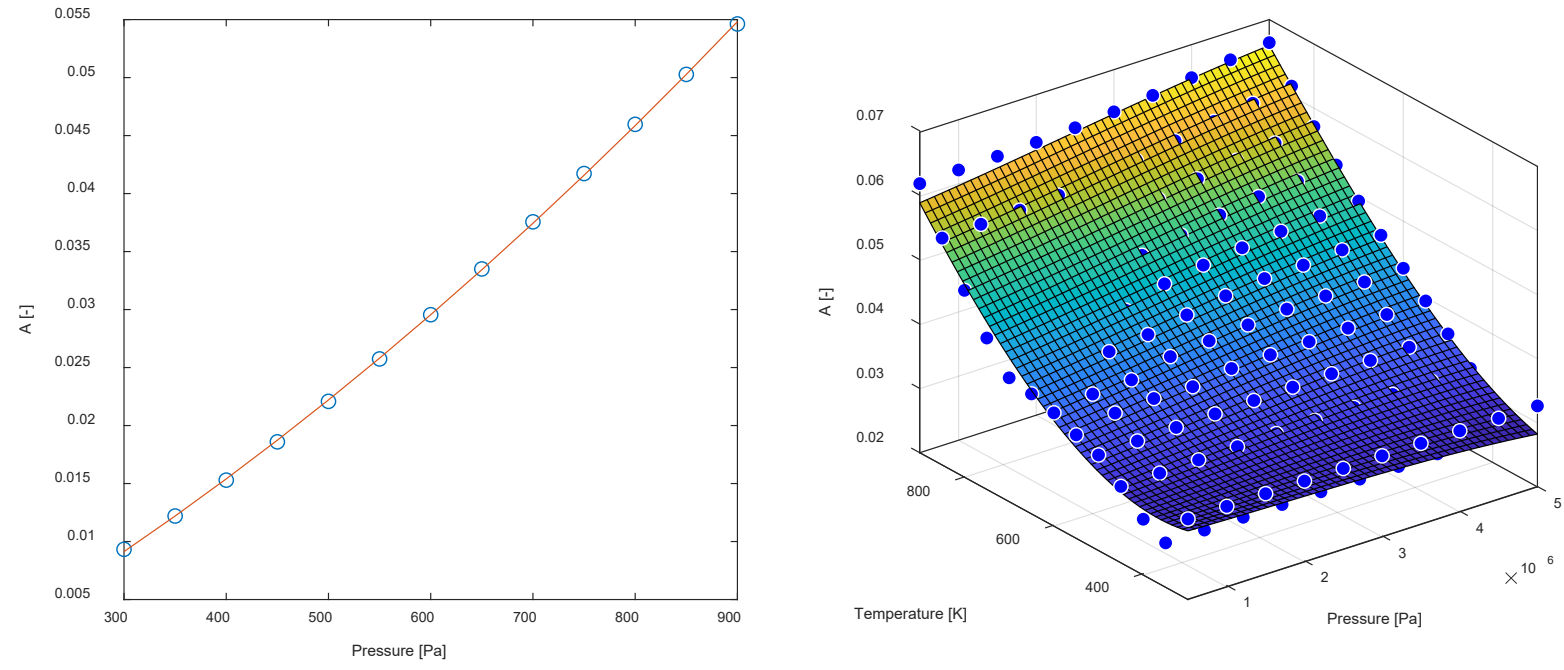

Figure 3: A comparison of a solely temperature-based function to a pressure-temperature surface

\section{Results}

To evaluate the effectiveness of the pressure-temperature surfaces in describing droplet vaporization behavior in a computationally efficient manner, the high-pressure DCM and CTM were run at a variety of temperatures and pressures. Jet-A fuel was used, which was represented as a mixture of 67 unique hydrocarbon species. The initial droplet radius was $50 \mu \mathrm{m}$ and the droplet initially existed solely in the liquid phase; this droplet was then exposed to a variety of temperature and pressure environments. The results of these tests are shown in Table 1 below. Gas thermal conductivity $\left(k_{g}\right)$, liquid thermal conductivity $\left(k_{l}\right)$, liquid viscosity $\left(\mu_{l}\right)$, gas diffusivity $\left(D_{g}\right)$, droplet surface temperature $\left(T_{s}\right)$, and droplet radius $(r)$ are shown. The last two, though not pressure-corrected properties, are included to evaluate whether the CTM still accurately described the physical behavior of the droplet.

Table 1: Average property errors various temperatures and pressures

\begin{tabular}{|c|c|c|c|c|c|c|c|}
\hline \multirow{2}{*}{$\begin{array}{c}\text { Pressure } \\
{[\mathrm{MPa}]}\end{array}$} & \multirow{2}{*}{$\begin{array}{c}\text { Temp } \\
{[\mathrm{K}]}\end{array}$} & \multicolumn{6}{|c|}{ Average error for CTM (compared to DCM) [\%] } \\
\cline { 3 - 8 } & $k_{g}$ & $k_{l}$ & $\mu_{l}$ & $D_{g}$ & $T_{s}$ & $r$ \\
\hline 1 & 500 & 1.68 & 1.20 & 8.93 & 6.01 & 0.10 & 15.89 \\
\hline 1 & 700 & 0.83 & 1.96 & 14.38 & 5.26 & 1.30 & 1.76 \\
\hline 2 & 600 & 1.48 & 1.07 & 15.72 & 5.97 & 0.19 & 4.07 \\
\hline 2 & 800 & 1.12 & 2.21 & 16.12 & 4.71 & 1.24 & 0.72 \\
\hline 5 & 600 & 1.03 & 1.23 & 20.42 & 4.02 & 0.17 & 2.74 \\
\hline 5 & 800 & 0.43 & 0.69 & 12.35 & 3.65 & 0.20 & 0.34 \\
\hline
\end{tabular}

The percent error shown is an average over time of the error between property values from the DCM and the CTM at each timestep of the simulation. As such, while helpful, high percent errors may be the result of noise in the data, as may be the case with liquid viscosity. While a smoothing procedure was implemented, some noise seems to have remained; Figure 4 provides a graphical comparison of the simulation results for a single scenario, in this case at $5 \mathrm{MPa}$ and $600 \mathrm{~K}$. 

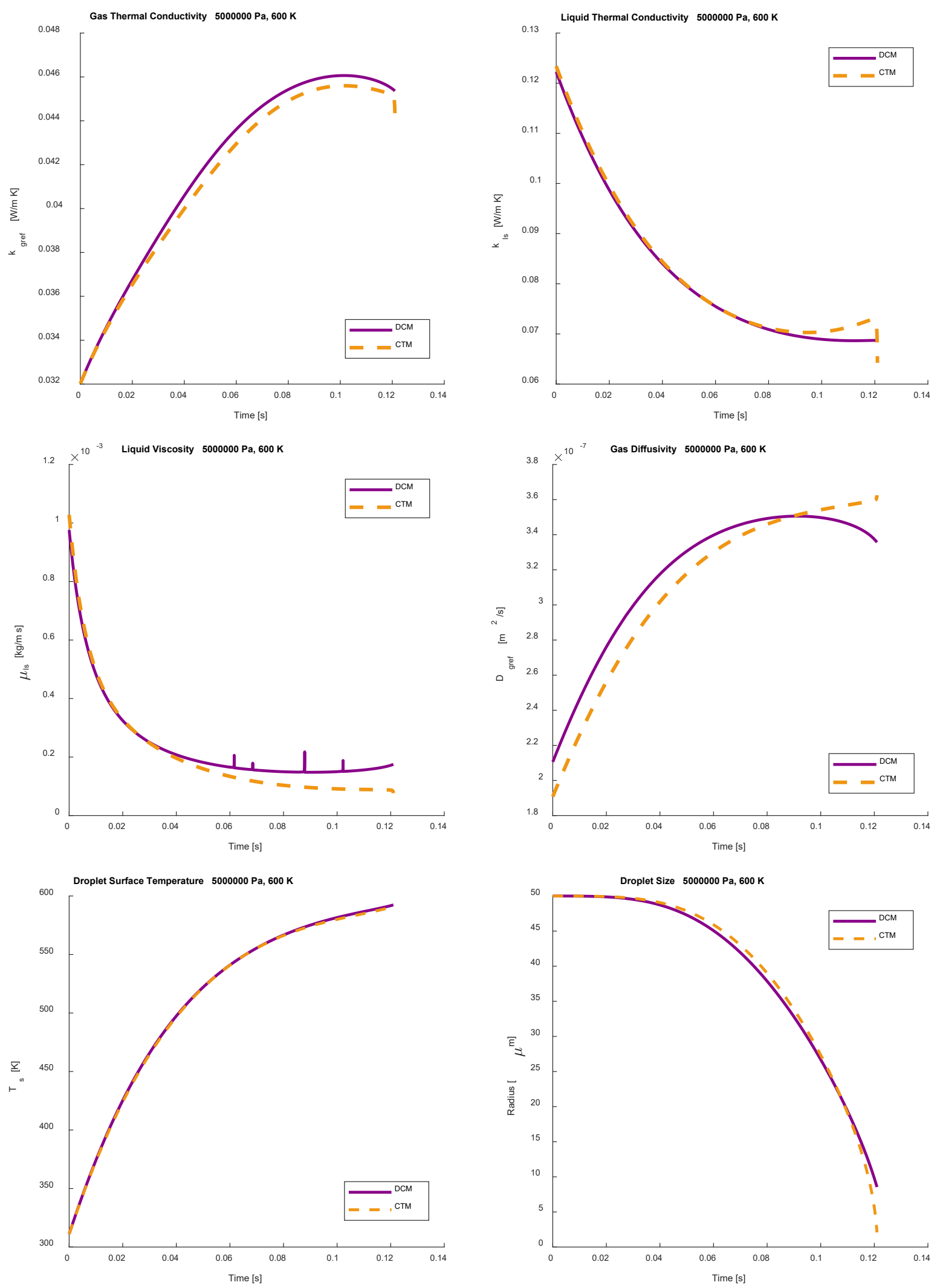

Figure 4: Droplet vaporization results at $5 \mathrm{MPa}$ and $600 \mathrm{~K}$ 


\section{Discussion}

The results suggest that the generated pressure-temperature surfaces are a promising method for adapting droplet vaporization CTMs for use at high pressures. In addition, the Takahashi method for adapting gas diffusivity appears to work well for both the discrete and continuous models. Of the five properties addressed in this paper, gas viscosity is the only property not able to be evaluated; limitations in the model used made it difficult to create a comparison between the DCM and CTM. For the rest of the properties, the small differences at high pressures seem promising, with liquid viscosity standing out as the only property to consistently register over $10 \%$ error. The success of the high-pressure CTM to replicate the droplet behavior - in the form of surface temperature and droplet radius - also points to the efficacy of these methods.

Table 2: Simulation runtimes of low and high-pressure models

\begin{tabular}{|c|c|c|c|c|}
\hline $\begin{array}{c}\text { Pressure } \\
{[\mathrm{MPa}]}\end{array}$ & $\begin{array}{c}\text { Temp } \\
{[\mathrm{K}]}\end{array}$ & $\begin{array}{c}\text { Low-pressure } \\
\text { DCM Runtime } \\
{[\mathrm{s}]}\end{array}$ & $\begin{array}{c}\text { Low-pressure } \\
\text { CTM Runtime } \\
{[\mathrm{s}]}\end{array}$ & $\begin{array}{c}\text { High-pressure } \\
\text { CTM Runtime } \\
{[\mathrm{s}]}\end{array}$ \\
\hline 1 & 500 & 3.7359 & 0.6943 & 0.7109 \\
\hline 2 & 600 & 3.8995 & 0.8472 & 0.8199 \\
\hline 5 & 600 & 3.9668 & 0.8449 & 0.8180 \\
\hline
\end{tabular}

Perhaps most importantly, the high-pressure CTM maintains its computational edge. As Table 2 shows, the CTM proposed in this paper has comparable runtimes to its low-pressure counterpart and still outperforms a low-pressure DCM as well. Due to a lack of optimization of the highpressure DCM with regards to minimizing computation time, it has been omitted from this comparison.

Further investigation is required to refine the approach presented in this paper. First, the droplet models were not always able to run to completion, suggesting that there may be some discrepancies in the code set that are restricting the models' calculations towards the end of a droplet's lifetime. In addition, while the chosen correction calculations fit well into this model and work to establish a proof-of-concept, more complex mixing rules may be required in order to more accurately depict fuel properties at high pressures. Such methods would require more comprehensive changes to the droplet model and may be a fruitful avenue for future research.

\section{References}

Cooney, A. Y., \& Singer, S. L. (2019). A hybrid droplet vaporization-chemical surrogate approach for emulating vaporization, physical properties, and chemical combustion behavior of multicomponent fuels. Proceedings of the Combustion Institute, 37(3), 3229-3236. doi:10.1016/j.proci.2018.07.071.

Ely, J. F., \& Hanley, H. J. M. (1983). Prediction of transport properties. 2. thermal conductivity of pure fluids and mixtures. Industrial \& Engineering Chemistry Fundamentals, 22(1), 90-97. doi:10.1021/1100009a016.

Fuller, E. N., Schettler, P. D., \& Giddings, J. C. (1966). new method for prediction of binary gas-phase diffusion coefficients. Industrial \& Engineering Chemistry, 58(5), 18-27. doi:10.1021/ie50677a007.

Jossi, J. A., Stiel, L. I., \& Thodos, G. (1962). The viscosity of pure substances in the dense gaseous and liquid phases. AIChE Journal, 8(1), 59-63. doi:10.1002/aic.690080116. 
Latini, G., \& Baroncini, C., (1983). Effects of temperature and pressure on organic liquid thermal conductivity. High Temperatures-High Pressures, 15, 407-411.

Lovestead, T. M., Burger, J. L., Schneider, N., \& Bruno, T. J. (2016). Comprehensive assessment of composition and thermochemical variability by high resolution GC/QToF- MS and the advanced distillation-curve method as a basis of comparison for reference fuel development. Energy \& Fuels, 30(12), 10029-10044. doi:10.1021/acs.energyfuels.6b01837.

Lucas, K. (1981). Die druckabhängigkeit der viskosität von flüssigkeiten - eine einfache abschätzung. Chemie Ingenieur Technik, 53(12), 959-960. doi:10.1002/cite.330531209.

Nomura, H., Ujiie, Y., Rath, H. J., Sato, J., \& Kono, M. (1996). Experimental study on high-pressure droplet evaporation using microgravity conditions. Symposium (International) on Combustion, 26(1), 1267-1273. doi:10.1016/S0082-0784(96)80344-4

Reid, R. C., Prausnitz, J. M., \& Poling, B. E. (1987). The properties of gases and liquids (Fourth ed.). New York: McGraw-Hill.

Tamim, J., \& Hallett, W. L. H. (1995). A continuous thermodynamics model for multicomponent droplet vaporization. Chemical Engineering Science, 50(18), 2933-2942. doi:10.1016/0009-2509(95)00131-N

Takahashi, S. (1974). Preparation of a generalized chart of the diffusion coefficients of gases at high pressures. Journal of Chemical Engineering of Japan, 7(6), 417-420.

Torres, D. J., O'Rourke, P. J., \& Amsden, A. A. (2003). A discrete multicomponent fuel model. Atomization and Sprays, 13(2-3), 42. doi:10.1615/AtomizSpr.v13.i23.10

Yan, C., \& Aggarwal, S. K. (2006). A high-pressure droplet model for spray simulations. Journal of Engineering for Gas Turbines and Power, 128(3), 482. doi:10.1115/1.1915390

Yaws, Carl L.. (2012; 2013; 2014). Yaws' Critical Property Data for Chemical Engineers and Chemists. Knovel. Retrieved from https://app.knovel.com/hotlink/toc/id:kpYCPDCECD/yaws-critical-property/yawscritical-property.

Zhang, L., \& Kong, S. (2011). High-pressure vaporization modeling of multi-component petroleum-biofuel mixtures under engine conditions. Combustion and Flame, 158(9), 1705-1717. doi:10.1016/j.combustflame.2011.01.002. 